\title{
Comentario a la sentencia de la Sala Constitucional número 2011-010832: declaratoria de inconstitucionalidad del $60 \%$ de apoyo exigido para la huelga legal
}

\author{
Mágister Fabián Arrieta Segleau \\ Licenciado en Derecho por la Universidad de Costa Rica, Magíster en Derecho del Trabajo y de la Seguridad Social por la \\ UNED. \\ Profesor de la Maestría de Derecho del Trabajo y de la Seguridad Social, UNED. \\ Juez civil y de trabajo del Tribunal del Segundo Circuito Judicial de la Zona Atlántica. \\ Correo electrónico: farrietas@poder-judicial.go.cr
}

Recibido: Enero 2015 • Aceptado: Febrero 2015

\begin{abstract}
RESUMEN
El presente trabajo constituye un análisis crítico de la sentencia de la Sala Constitucional de la Corte Suprema de Justicia número 2011-010832. Esta anuló la exigencia de un porcentaje de apoyo del $60 \%$ para la declaratoria de legalidad de la huelga. Concretamente se examina las consecuencias de ese fallo en la flexibilización del ejercicio de ese derecho fundamental, en su naturaleza jurídica, y regulación procesal. Se hace referencia a la situación actual del requisito cuantitativo para asistir a la huelga, a partir del pronunciamiento del Tribunal Constitucional y las resoluciones de los órganos de control de la OIT, particularmente la Comisión de Expertos en la Aplicación de Convenios y Recomendaciones, y cuál es la labor de los Tribunales de Trabajo, ante la delegación efectuada en el voto a la hora de determinar el quórum exigido para la declaratoria de huelga legal.

También se aborda la problemática provocada por el fallo al establecer que, previo a la declaratoria de ilegalidad y durante la vigencia del movimiento de presión cuando éste sea considerado legal, se debe mantener el pago de salario para las personas que huelgan. Además, se fundamenta la incidencia de esa determinación en la naturaleza jurídica de ese instituto jurídico y las posibles repercusiones prácticas para la empresa. Finalmente, se hace referencia a la participación en el movimiento de presión como justa causa de despido y la naturaleza del incidente de calificación de huelga y su repercusión en la terminación de los contratos de trabajo.
\end{abstract}

Palabras clave: Huelga, porcentaje, flexibilización, referéndum, libertad sindical.

\section{ABSTRACT}

The present article is a critical analysis about sentence number 2011-010832 from Constitutional Chamber of Supreme Court of Justice. This sentence annulled the requirement of a percentage of the $70 \%$ support for the declaration of the legality of the strike. Specifically examines the consequences of that ruling in the easing of the exercise of this fundamental right, in their legal nature, and procedural regulation. Reference is made to the current situation of the quantitative requirement to attend the strike, based on the ruling of the Constitutional Court and the resolutions of the supervisory bodies of the ILO, particularly the Committee of Experts on the Application of Conventions and Recommendations, and what is the work of the Labour Courts, before the delegation carried out in vote in determining the quorum required for the declaration of legal strike. 
Also addresses the problems caused by the failure to establish, prior to the declaration of illegality, and during the term of the industrial action when this is considered legal, should be the payment of wages for people who calls, the incidence of that determination on the legal nature of that legal institute and the possible practical implications for the company. Finally, refers to the participation in the movement of pressure as just cause for dismissal and the nature of the incident of qualification of strike and its impact on the termination of contracts of employment.

Key words: Strike, percentage, easing, referendum, freedom of association.

\section{Introducción}

El instituto de la huelga legal en Costa Rica es como una nebulosa oscura: su presencia se detecta únicamente leyendo el Código de Trabajo a contraluz; o en su defecto, delirando con los alcances que algún precepto constitucional pueda tener sobre la realidad social en la que se desenvuelven las relaciones de trabajo.

En el intento de explicar las razones que motivan esa paradigmática forma de ocultar lo evidente (que las relaciones de trabajo son conflictivas y que requieren una válvula de escape), probablemente se encontrarán múltiples explicaciones. Cada una de ellas relacionadas con el desarrollo histórico de la sociedad costarricense, pero que redundan en un enraizado temor (dentro de los sectores económicamente dominantes) de permitir toda forma de expresión de acción colectiva de la clase trabajadora.

Esa realidad histórica y social se ve reflejada en el desarrollo legal que ha tenido el derecho de huelga en Costa Rica. Este es instituido en el Código de Trabajo costarricense desde el año 1943. En su regulación sustancial no ha tenido reformas legales trascendentales desde los años 70 , y en definitiva, ninguna con el objetivo de flexibilizar su ejercicio.

Ante ese panorama la clase trabajadora y sus organizaciones sociales se han visto obligadas a acudir a la Sala Constitucional, a través de la vía de la acción de inconstitucionalidad, para tratar de erradicar del ordenamiento jurídico una serie de disposiciones legales que históricamente han tornado impracticable la huelga legal.

En este trabajo no se pretende abordar cada una de esas reinterpretaciones constitucionales que han modificado sustancialmente los alcances del derecho de huelga en Costa Rica, ya que ello debe ser objeto de un estudio más profundo que involucre un análisis integral del fenómeno jurídico, pero sí detenerse en la valoración de la sentencia de la Sala Constitucional número 2011-010832, de las 14 horas y 30 minutos del 12 de agosto de 2011, cuya incidencia en la naturaleza jurídica de ese instituto aún no termina de dimensionarse adecuadamente dada su emisión relativamente reciente.

Particularmente interesa hacer referencia a su requisito cuantitativo (el porcentaje de apoyo), su incidencia en los aspectos procesales de la huelga y su trámite de calificación; a la modificación de su naturaleza jurídica (¿o su desnaturalización?), en virtud de la obligación (definida en el fallo) de cancelar los salarios de las personas que permanezcan holgando; a la limitación a la potestad patronal de concluir los contratos de trabajo de quienes formen parte del movimiento de presión (previo a la declaratoria de ilegalidad) y las repercusiones de esa restricción en la tramitación del incidente de calificación de huelga, particularmente cuando esta ha cesado.

\section{La determinación del porcentaje de apoyo a la huelga}

La sentencia de comentario tuvo como génesis una acción de inconstitucionalidad promovida por una organización sindical contra los artículos 373, incisos b) y c), y 377 del Código de Trabajo: el primero exige el agotamiento del procedimiento de conciliación previa y establecía (antes de su anulación) como requisito para la huelga legal el $60 \%$ de apoyo de los trabajadores de la empresa, lugar o negocio del que se tratare; y el segundo, en cuanto establece la posibilidad de extinguir, sin responsabilidad patronal, los contratos de trabajo de las personas huelguistas. 
Como argumento para sustentar la acción, se adujo que los requisitos definidos en el ordinal 373 citado imposibilitan en la práctica el ejercicio de la huelga legal; mientras que el numeral 377 representa un freno adicional; ya que, ante la imposibilidad de cumplir con los presupuestos para ejercitar legalmente el movimiento de presión, las personas trabajadoras se ven expuestas a la pérdida de su empleo, sin que se garantice su derecho al debido proceso; por lo que se argumentó violados los artículos 41 y 61 constitucionales.

La Sala Constitucional, por mayoría de sus votos, anuló el requisito del $60 \%$ hasta esa fecha exigido para la declaratoria de la huelga legal, por lo que se dejó incólume la obligación de acudir al proceso de conciliación reglado en el Capítulo III del Título Sétimo del Código de Trabajo. Respecto a la exigencia cuantitativa de apoyo, adujo que el porcentaje requerido por el ordenamiento jurídico costarricense "excede el límite de lo que la OIT ha calificado como razonable para ser un requisito legítimo y que no impida el ejercicio del derecho de huelga" (Sala Constitucional, voto 2011-010832, 12 de agosto de 2011) . Luego la Sala, en una lectura personal de lo que han expresado los órganos de control de esa organización internacional, refirió que "sí debe exigirse un porcentaje determinado, una mayoría, como lo indica la OIT" (Sala Constitucional, voto 2011010832, 12 de agosto de 2011), estableciendo la obligación de la Asamblea Legislativa de fijar ese nuevo número "que no debe ser excesivo de manera que impida el ejercicio del derecho de huelga". En el ínterin, dejó en manos de "los jueces de la República que les corresponde conocer de este tipo de asuntos (...) aplicar los convenios y las recomendaciones del Comité de la OIT para determinar el porcentaje correspondiente" (Sala Constitucional, voto 2011-010832, 12 de agosto de 2011).

A partir de aquí la sentencia admite varios comentarios. En primer término, se considera, con el respeto que el Alto Tribunal Constitucional se merece, que en el análisis que le permite arribar a la conclusión de que la norma es inconstitucional malinterpreta lo que es el fenómeno colectivo de las relaciones laborales, ya que lo asocia a un aspecto meramente cuantitativo. Lo anterior, a pesar de que se sabe en la doctrina laboralista, desde su origen, que lo que caracteriza a ese tipo de conflictos no es el número de los participantes (aunque la participación de un colectivo interesa), sino que las pretensiones estén relacionadas con reivindicaciones de carácter económico-sociales o, en todo caso, con intereses propios del colectivo de personas trabajadoras (entendida la huelga en un sentido amplio y flexible). ${ }^{1}$

De allí que, sostener que la OIT ha establecido que se deba exigir necesariamente un quórum determinado de apoyo a la huelga resulta impreciso. Los órganos de control (particularmente el Comité de Libertad Sindical) se han limitado a examinar, en casos concretos, distintos ordenamientos jurídicos donde se exige un porcentaje de apoyo al movimiento para su legalidad. Estos concluyen que establecer una participación de dos terceras partes, más de un $50 \%$ o mayoría absoluta, puede significar una exigencia difícil o imposible de cumplir y en consecuencia contraria al Convenio sobre la libertad sindical y la protección del derecho de sindicación, 1948 (núm. 87). A pesar de esto, el Comité nunca se ha expresado en el sentido de que deba establecerse necesariamente ese porcentaje, y tampoco ha dictado una resolución admitiendo como válida la exigencia obligatoria de alguna mayoría de apoyo. ${ }^{2}$

Más precisa, en cuanto a este tema, resulta la posición de la Comisión de Expertos en la Aplicación de Convenios y Recomendaciones cuando refiere:

La legislación de muchos países subordina el ejercicio del derecho de huelga a la aprobación previa de esa acción por un cierto porcentaje de trabajadores. Esta exigencia no plantea, en principio, ningún problema respecto al Convenio [núm. 87], pero las modalidades de escrutinio, el quórum y la mayoría exigida no deberían ser tales que el ejercicio del

1. En particular sobre la flexibilización del derecho de huelga, resulta de gran utilidad ver Ermida, 1999.

2. Al respecto, se puede revisar: Gernigon, Odero y Guido, 2000: 30 . 
derecho de huelga resultase, en la práctica, muy dificil, e incluso imposible.

Las condiciones establecidas en las diversas legislaciones varían enormemente, y su compatibilidad con el Convenio puede depender también de elementos concretos, tales como la diseminación o el alejamiento geográfico de los centros de trabajo, o incluso la estructura de la negociación colectiva (por empresa o por industria):

[...] Si un Estado Miembro considera adecuado prever en su legislación disposiciones que exijan que las acciones de la huelga deban ser votadas por los trabajadores, dicho Estado deberá asegurar que sólo se tomen en consideración los votos emitidos, y que el quórum o la mayoría se fije en un nivel razonable [...]. (Gernigon, Odero y Guido, 2000: 31)

De una lectura reposada de los anteriores pronunciamientos, se debería concluir en este apartado que la exigencia que le fijó la Sala Constitucional al Poder Legislativo primero, y a los jueces y a las juezas de trabajo mientras tanto, de definir obligatoriamente un porcentaje de apoyo para considerar legal la huelga, va más allá de cualquier interpretación que los Órganos de Control de la OIT hayan efectuado del Convenio 87. Sin embargo, partiendo de la premisa de que el fallo constitucional resulta obligatorio erga omnes (artículo 13 de la Ley de la Jurisdicción Constitucional), la determinación del requisito numérico de participantes deberá efectuarse de conformidad con lo expresado por la Comisión de Expertos. Esto en el sentido de que, en primer lugar, se deberán observar las particularidades que envuelven el ámbito en el que se desarrolla la huelga (el tipo de empresa, la cantidad de personal, la rama de actividad, la ubicación geográfica de los centros de trabajo, si es una huelga sindical o de una coalición temporal, los objetivos del movimiento, y cualquier otro dato de interés que pueda dimensionar la realidad del conflicto), para definir un porcentaje que se adecúe a la realidad del caso concreto; y en segundo lugar, como un factor clave para proteger al personal de posibles represalias, en la determinación del quórum se deberán considerar únicamente a las personas que participen del referéndum, es decir, el porcentaje de apoyo. A este deberá extraerse de quienes voten, sin considerar a quienes se abstienen de participar, luego de un proceso secreto de consulta donde existan verdaderas garantías para la expresión libre de la voluntad.

La forma propuesta de efectuar ese trámite tendría como finalidad minimizar la posibilidad de que la entidad patronal se valga de la amenaza y la intimidación para desestimular la participación de las personas trabajadoras en el referéndum. Lo que se pretende es que se estimule la concurrencia del personal a las votaciones, redundando en un fortalecimiento de la libertad sindical.

\section{La desnaturalización de la huelga en sentido estricto}

Pocas veces se tiene la oportunidad de presenciar una transformación tan radical de un instituto jurídico, como el sufrido por la huelga a partir de la emisión de la sentencia de comentario. La Sala Constitucional, a pesar de no formar parte de los motivos de inconstitucionalidad esbozados por el sindicato recurrente, interpretó el numeral 377 del Código de Trabajo en el sentido que:

(...) la terminación de los contratos de trabajo es a partir de la declaratoria de ilegalidad de la huelga $y$, en consecuencia, los trabajadores que participaron en el movimiento huelguístico antes de esa declaratoria no pueden ser despedidos, rebajados sus salarios ni sancionados de forma alguna por la mera participación en la huelga, pues constituye una limitación desproporcionada e irrazonable al ejercicio de un derecho fundamental, lo que afecta su contenido esencial. (Sala Constitucional, voto 2011010832, 12 de agosto de 2011).

El mandato de la Sala es categórico, no es posible descontar el salario al personal que participe de la huelga en el tanto ésta no sea declarada ilegal. Lo anterior, como es lógico, no ocurriría hasta que el juez o la jueza de trabajo efectúe ese pronunciamiento dentro del respectivo incidente de calificación. Ello implica que ante el supuesto de huelga legal, la parte patronal estaría siempre obligada a 
pagar el salario a quienes participen del movimiento de presión.

Lo anterior, según el criterio del autor del presente escrito, contraviene la naturaleza misma de la huelga, entendida como un abandono temporal del trabajo, que trae como consecuencia la suspensión de los contratos de trabajo vigentes en la empresa, lugar o negocio en que se declare ${ }^{3}$. Siendo la huelga una suspensión del contrato de trabajo, inmediatamente las principales prestaciones derivadas de éste cesan, a saber: la obligación de trabajar y la obligación de pagar el salario. (Pasco, 1997)

Los efectos jurídicos de la suspensión de los contratos de trabajo, en relación con el tema de la huelga, tienen una especial trascendencia para su adecuado desenvolvimiento. El objetivo de estos movimientos de presión es reivindicar la posición del colectivo ${ }^{5}$ obrero frente algún tema de interés laboral, no la aniquilación de la empresa. Se reconoce la posibilidad de encausar el conflicto a través del mecanismo de acción directa, infligiendo un daño a la entidad patronal (con motivo de la parálisis de la producción), a cambio de sufrir (en principio) una afectación en los ingresos. En definitiva la huelga se concibe como un pulso entre la coalición del personal y la empresa, donde en principio triunfará el que esté mejor preparado para soportar sin percibir ganancias o ingresos durante la prolongación del conflicto ${ }^{6}$.

Desde esa perspectiva, se considera que una de las consecuencias prácticas del voto de la Sala es la de desnaturalizar los efectos tradicionales de la huelga. Esta genera un desbalance en perjuicio de la

3. Ver al respecto los artículos 371 y 372 del Código de Trabajo. (Código de Trabajo, Ley ${ }^{\circ} 2$ de 26 de agosto de 1943).

4. En referencia al cese de la obligación de pagar el salario, la doctrina iuslaboralista patria ya ha hecho referencia, al expresar: "La principal consecuencia de la suspensión de los contratos de trabajo respecto de los trabajadores es la falta de percepción del salario; sin embargo, como principio general que es, admite excepciones, y la legislación costarricense las contempla en el artículo 371 [actual 388 del Código de Trabajo] (...)" (Van der Laat, 1979: 126).

5. Sobre este tema ver: (Sala, 2002: 390).

6. Sobre este tema ver: (Sala, 2002: 447). parte patronal, al verse obligada a soportar el pago del salario de todo su personal, aún sin producción, mientras se lleva a cabo la calificación de legalidad de la huelga y, en caso de ser declarada legal, durante todo el tiempo que esta se mantenga, con el consecuente riesgo para la viabilidad económica de la empresa.

Esa situación conlleva a que la sentencia constitucional haya modificado el numeral 372 del Código de Trabajo, suponiendo para la huelga ya no una suspensión de los contratos de trabajo, sino únicamente de la obligación de trabajar. También varió los supuestos de "huelga justa", contemplados en el artículo 378 ídem, extendiéndolos a todos los casos de huelga legal.

\section{Las restricciones al despido y el incidente de calificación de huelga}

Otro aspecto que la Sala Constitucional impuso en el voto número 2011-010832, es la prohibición de despedir a los huelguistas por su mera participación en el movimiento, previo a que exista una declaratoria de ilegalidad. En cuanto a este punto se considera que la Sala viene a reforzar un aspecto de mucha importancia: la posibilidad de despedir al personal que se suma a un movimiento de huelga no surge únicamente por la acción de participar en dicho evento, ya que ello contravendría el ejercicio de un derecho fundamental, sino a partir de que exista una declaratoria judicial de ilegalidad. De esta forma debe entenderse que la causal de terminación del contrato de trabajo, sin responsabilidad patronal

7. En doctrina, la huelga justa se conoce como aquel movimiento originado en incumplimientos graves de la parte patronal que justifican el estado de huelga y que como consecuencia permiten que las personas que se suman al movimiento de presión, posteriormente, cobren los sueldos caídos, y que en nuestro ordenamiento apare regulada en el 378, que señala: "Si los Tribunales de Trabajo declaran que los motivos de una huelga legal son imputables al patrono, por incumplimiento del contrato o contratos de trabajo, por negativa injustificada a celebrar una convención colectiva o por maltrato o violencia contra los trabajadores, condenarán a aquél al pago de los salarios correspondientes a los días en que éstos hayan holgado." 
tiene su origen en esa causa objetiva y su posterior comunicación al personal para que se reintegre a las labores, con la consecuente negativa de este a cumplir con esa orden. ${ }^{8}$

Esto aclara una situación que, desde la óptica procesal del tratamiento de la huelga, puede generar algún problema (sobre todo en la praxis judicial) y que tiene que ver con el incidente de calificación del movimiento y su tramitación luego de que este ha concluido.

Un primer aspecto que hay que definir es cuál es el propósito para llevar a cabo la calificación de la huelga. En referencia a ese tema, el ordenamiento jurídico costarricense es claro: la declaratoria de ilegalidad conlleva, una vez que ha sido comunicada a las personas trabajadoras, la posibilidad de terminar los contratos con los huelguistas que no se reincorporen al trabajo (artículo 377 del Código de Trabajo, Ley $n^{\circ} 2$ de 26 de agosto de 1943), y la obligación para los Tribunales de Trabajo de ordenarle a las autoridades de policía que garanticen la continuidad de las labores (artículo 388 el Código de Trabajo, Ley $n^{\circ} 2$ de 26 de agosto de 1943).

En consecuencia, una vez concluido el movimiento de huelga por las razones que sea (y por ende reiniciadas las labores), ya no tiene ningún sentido práctico continuar con el trámite de calificación de huelga, dado que no podría el empleador aplicar la causal de terminación del contrato del 377 y resultaría insulso ordenar la continuidad de unas labores que ya se están ejecutando. Por esto, el incidente (que por tener esa naturaleza ya de por sí requiere de la existencia de una huelga para subsistir) debería ser archivado, desde luego, sin perjuicio de que en caso de que se reinicie el mismo movimiento (tomando en cuenta que se produzca en un corto periodo y por las mismas causas), nada impediría que interponga una nueva incidencia y se continúe con la calificación de la huelga para los efectos antes mencionados.

Finalmente, cuando el empleador decida terminar el contrato de trabajo con los huelguistas, una vez concluido el movimiento, independientemente

8. Ver sobre este particular: Van der Laat, 1979: 143-147. de la causal alegada, siempre será posible discutir en el proceso ordinario laboral si la decisión patronal obedeció a un acto de represalia tendiente a restringir el ejercicio pleno de la libertad sindical, y por ende, discriminatorio. En caso de que esto se presentara, se podría dar pie a una declaratoria de nulidad del despido, con la consecuente orden de reinstalación y pago de salarios caídos que puedan resultar procedentes (artículo 363 del Código de Trabajo) y demás indemnizaciones por daños y perjuicios que se puedan reclamar en esa vía. Siempre en el entendido que no podrá ser imputada como justa causa de despido, la sola participación de las personas trabajadoras en un movimiento de huelga que pudo no ser legal (por ausencia de algún requisito formal), si esta no fue declarada como tal durante su desarrollo, a través del incidente respectivo.

\section{Conclusiones}

El voto número 2011-010832 de la Sala Constitucional representa un pequeño avance en el camino de la flexibilización del derecho de huelga, al declarar inconstitucional la exigencia porcentaje de apoyo $60 \%$, que a todas luces resultaba desproporcionado e impedía en la práctica el ejercicio pleno de ese derecho. Sin embargo, sus efectos son limitados, en el tanto insta al Poder Legislativo a establecer un nuevo porcentaje mínimo de participación (pero menor al anterior), y obliga, mientras tanto, a los Tribunales de Trabajo a fijarlo en cada caso concreto.

Esa posición de la Sala, al considerar que el instituto de la huelga debe estar sometido a requisitos y restricciones de esa naturaleza para permitir su desarrollo, desconoce que, como modernamente se señala:

(...) la especificidad de la democracia, hoy, consiste en la legitimación del conflicto y el rechazo a eliminarlo por medio de la incorporación de un orden autoritario, a partir del reconocimiento de que él es el resultado natural e inevitable del pluralismo constitutivo del orden democrático. (Ermida, 1999: 13)

Por otro lado, la Sala incursionó en aspectos que no formaron parte de los reclamos del sindicato 
recurrente. Esto modificó la naturaleza jurídica de la huelga, al señalar que la suspensión de los contratos de trabajo no conlleva la consecuente liberación para la parte patronal de la obligación de pagar salarios. Esta variación estableció un desbalance inadecuado entre las partes del conflicto, que puede, incluso, poner en riesgo la viabilidad económica de la empresa y su continuidad como fuente de trabajo y de riqueza.

Por último, la Sala terminó de aclarar una vieja discusión que existía en torno a la participación de las personas trabajadoras dentro de un movimiento de huelga que pudiera considerarse eventualmente ilegal por no cumplir con todos los requisitos formales, al disponer que solo es posible despedir al personal que no se reincorpore a sus labores una vez que se ha declarado su ilegalidad. Esta resolución hizo más plausible que el trámite del incidente de calificación de huelga deba llevarse a cabo en el tanto el movimiento se mantenga vigente, y una vez finalizado el mismo, procede su archivo -si aún no se ha resuelto-, pudiendo interponerse nuevamente en la eventualidad de que el mismo movimiento se reactive.

\section{Bibliografía}

Costa Rica, Código de Trabajo, Ley n ${ }^{\circ} 2$ de 26 de agosto de 1943. Publicada en La Gaceta ${ }^{\circ} 192$ del 29 de agosto de 1943.

Costa Rica, Sala Constitucional de la Corte Suprema de Justicia. Voto 2011-010832, de las catorce horas y treinta minutos del doce de agosto de dos mil once.

Ermida, Oscar (1999). La Flexibilización de la Huelga. Montevideo: Fundación de Cultura Universitaria.

Gernigon, Bernard. Odero, Alberto; Guido, Horacio (2000). Principios de la OIT sobre el derecho de huelga. Ginebra: Oficina Internacional del Trabajo.

Pasco, Mario (1997). Suspensión del Contrato de Trabajo. En Néstor De Buen y Emilio Morgado, Instituciones de Derecho del Trabajo y de la Seguridad Social (págs. 481500). México: Universidad Autónoma de México.

SAla, Tomás (2002). Derecho Sindical. Valencia: Tirant lo blanch.

VAN Der LAAT, Bernardo (1979). La Huelga y el Paro en Costa Rica. San José: Juricentro. 
